\title{
Adaptive Generalized Predictive Control of a Heat Exchanger Pilot Plant
}

\author{
Zohra Zidane, ${ }^{1, *}$, Mustapha Ait Lafkih ${ }^{2}$, Mohamed Ramzi ${ }^{2}$ \\ ${ }^{1}$ Laboratory of Automatic and Energy Conversion (LAEC) Electrical Engineering Department, Faculty of Sciences and Technology \\ University of Sultan Moulay Slimane, B.P: 523 23000, Beni-Mellal, Morocco \\ ${ }^{2}$ Electrical Engineering Department, Faculty of Sciences and Technology University of Sultan Moulay Slimane, B.P: 523 23000, \\ Beni-Mellal, Morocco
}

\begin{abstract}
In this paper, the Adaptive Generalized Predictive Control is designed to control a heat exchanger pilot plant. The standard Generalized Predictive Control (GPC) algorithm is presented. The Adaptive Generalized Predictive Control is then applied to achieve set point tracking of the output of the plant. A Single Input Single Output (SISO) model is used for control purposes. The model parameters are estimated on-line using an identification algorithm based on Recursive Least Squares (RLS) method. The performance of the proposed controller is illustrated by a simulation example of a heat exchanger pilot plant. Obtained results demonstrate the effectiveness and superiority of the proposed algorithm.
\end{abstract}

Keywords Adaptive Control, Generalized Predictive Control, Heat Exchanger Pilot Plant, Parameter Estimator

\section{Introduction}

The Generalized Predictive Control (GPC) is one of the most favorite predictive control methods, popular in industry and also at universities. It was first published in 1987[1],[2]. The authors wanted to find one universal method to control different systems. GPC has been successfully implemented in many industrial applications, showing good performance and a certain degree of robustness. It is applicable[3] to the systems with non-minimal phase, unstable systems in open loop, systems with unknown or varying dead time, systems with unknown order and nonlinear systems approximated by linear models.

The basic idea of GPC[4],[5] is to calculate a sequence of future control signals in such a way that it minimizes a multistage cost function defined over a prediction horizon. The index to be optimized is the expectation of a quadratic function measuring the distance between the predicted system output and some reference sequence over the horizon plus a quadratic function measuring the control effort. The predictive model is carried out based on the solving Diophantine equations.

In the present paper the Adaptive Generalized Predictive Control method is designed to control a heat exchanger pilot plant and a Single Input Single Output (SISO) model is used for control purposes. The model parameters are estimated

* Corresponding author:

zidane.zohra@gmail.com (Zohra Zidane)

Published online at http://journal.sapub.org/jmea

Copyright (C) 2012 Scientific \& Academic Publishing. All Rights Reserved
On-line using an identification algorithm based on Recursive Least Squares method. It is proved in the paper that in spite of important variations of the plant output; the developed adaptive structure maintains high level of performances (tracking, disturbance robustness and overshoot, cancellation of oscillation).

The paper is organized as follows. Section II presents the Generalized Predictive Control algorithm. Section III is devoted the description of the adaptive control algorithm. In section IV, the effectiveness and superiority of the adaptive system, is demonstrated by simulation example. Some concluding remarks end the paper.

\section{Generalized Predictive Control Algorithm}

The GPC scheme[6] can be seen in Figure 1. It consists of the plant to be controlled, a reference model that specifies the desired performance of the plant, a linear model of the plant, and the Cost Function Minimization (CFM) algorithm that determines the input needed to produce the plant's desired performance. The GPC algorithm consists of the CFM block.

The GPC system starts with the input signal, $r(t)$, which is presented to the reference model. This model produces a tracking reference signal, $w(t)$ that is used as an input to the CFM block. The CFM algorithm produces an output, which is used as an input to the plant. Between samples, the CFM algorithm uses this model to calculate the next control input, $\mathrm{u}(\mathrm{t}+1)$, from predictions of the response from the plant's model. Once the cost function is minimized, this input is passed to the plant. This algorithm is outlined below. 


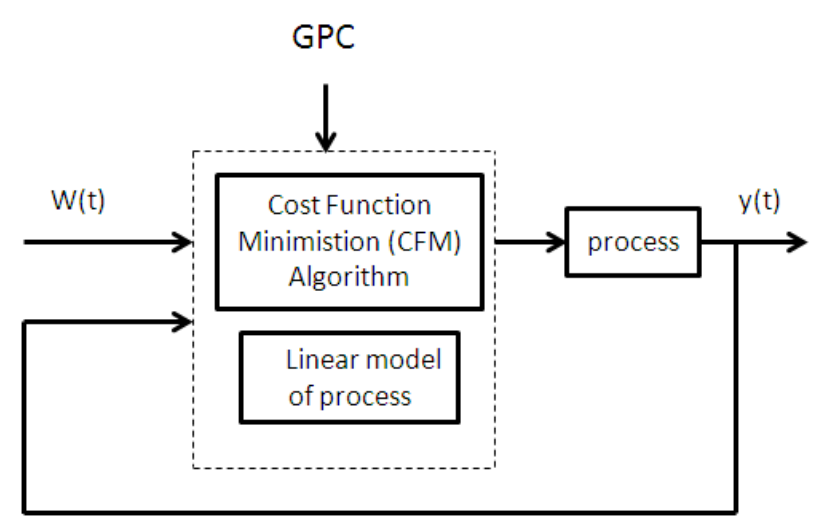

Figure 1. Basic structure of GPC

When considering regulation about a particular operating point, even a non-linear plant generally admits a locally-linearized model[1] and[2]:

$$
A\left(q^{-1}\right) y(t)=B\left(q^{-1}\right) u(t-1)+w(t)
$$

Where, $\mathrm{A}$ and $\mathrm{B}$ are polynomials in the backward shift operator $q^{-1}$ :

$$
\begin{aligned}
& A\left(q^{-1}\right)=1+a_{1} q^{-1}+\ldots+a_{n a} q^{-n a} \\
& B\left(q^{-1}\right)=b_{0}+b_{1} q^{-1}+\ldots+b_{n b} q^{-n b}
\end{aligned}
$$

If the plant has a non-zeros dead-time the leading elements of the polynomial $B\left(q^{-1}\right)$ are zero.

Where:

$\mathrm{u}(\mathrm{t})$ is the control input.

$y(t)$ is the measured variable or output.

$\mathrm{w}(\mathrm{t})$ is a disturbance term.

In literature $w(t)$ has been considered to be a moving average form:

$$
w(t)=C\left(q^{-1}\right) \xi(t)
$$

Where: $C\left(q^{-1}\right)=1+c_{1} q^{-1}+\ldots+c_{n c} q^{-n c}$

In this equation $\xi(t)$ is uncorrelated random sequence, and combining with (1) we obtain the CARMA (Controlled Autoregressive Moving Average):

$$
A\left(q^{-1}\right) y(t)=B\left(q^{-1}\right) u(t-1)+C\left(q^{-1}\right) \xi(t)
$$

Where, $\Delta$ is the differencing operator $1-q^{-1}$ is the back shift operator such that $q^{-1} y(t)=y(t-1)$. For simplicity in the here $C\left(q^{-1}\right)$ is chosen to be 1 .

The objective of the GPC control is the output $y(t)$ to follow some reference signal $y *(t)$ taking into account the control effort. This can be expressed in the following cost function: locally-linearized model[1] and[2]:

$$
\begin{aligned}
& J\left(h_{i}, h_{p}, h_{c}, t\right)= \\
& E\left\{\sum_{h_{i}}^{h_{p}}\left[y(t+j)-y^{*}(t+j)\right]^{T} R\left[y(t+j)-y^{*}(t+j)\right]\right. \\
& \left.+\sum_{h i}^{h_{C}} \Delta u^{T}(t+j-1) Q \Delta u(t+j-1)\right\}
\end{aligned}
$$

Where: $\mathrm{h}_{\mathrm{p}}$ is the prediction horizon.

$\mathrm{h}_{\mathrm{i}}$ is the initial horizon.

$\mathrm{h}_{\mathrm{c}}$ is the control horizon.

$y^{*}(t)$ is the output reference.

$\mathrm{R}$ is the output weighting factor.

$\mathrm{Q}$ is the control weighting factor.

The control objective is to compute at each time $t$, control inputs that minimize the quadratic criterion $J\left(h_{i}, h_{p}, h_{c}, t\right)$ for this there are two cases:

- Case $1 h_{c}=h_{p}, h_{i}=1$

Let us first build $\mathrm{j}$-step ahead predictors with following Diophantine equation:

$$
\begin{gathered}
1=E^{j}\left(q^{-1}\right) A\left(q^{-1}\right) \Delta\left(q^{-1}\right)+q^{-j} F^{j}\left(q^{-1}\right) \\
j=1 \ldots h p
\end{gathered}
$$

Where:

$$
\begin{aligned}
& E^{j}\left(q^{-1}\right)=1+e_{1} q^{-1}+\ldots+e_{j-1} q^{-(j-1)} \\
& F^{j}\left(q^{-1}\right)=f_{0}^{j}+f_{1}^{j} q^{-1}+\ldots+f_{n a}^{j} q^{-n a}
\end{aligned}
$$

The polynomials $E^{j}\left(q^{-1}\right)$ and $F^{j}\left(q^{-1}\right)$ are uniquely defined by: $A\left(q^{-1}\right), \Delta\left(q^{-1}\right)$ and $\mathrm{j}$.

Using equation (1) and (5) we obtain:

$$
\begin{aligned}
y(t+j)= & E^{j}\left(q^{-1}\right) B\left(q^{-1}\right) \Delta u(t+j-1)+F^{j}\left(q^{-1}\right) y(t) \\
& +E^{j}\left(q^{-1}\right) \xi(t+j)
\end{aligned}
$$

The optimal predictor, given measured output data up to time $t$ and given $u(t+i)$ for $i>1$, is clearly:

$$
\hat{y}(t+j / t)=G^{j}\left(q^{-1}\right) \Delta u(t+j-1)+F^{j}\left(q^{-1}\right) y(t)
$$

Where, $G^{j}\left(q^{-1}\right)=E^{j}\left(q^{-1}\right) B\left(q^{-1}\right)$

Defining $\quad G^{j}\left(q^{-1}\right)=g_{0}^{j}+g_{1}^{j} q^{-1}+\ldots+g_{j-1}^{j} q^{-(j-1)}$ then the equation above can be written in the key vector form:

$$
\hat{Y}=G \Delta U_{t}+Y_{0}
$$

Where the vectors are all $h_{p} \times 1$ :

$$
\begin{aligned}
\hat{Y^{T}} & =\left[\begin{array}{l}
\hat{y}^{T}(t+1) \ldots \ldots \ldots \ldots . \hat{y}\left(t+h_{p}\right) \\
T
\end{array}\right] \\
\Delta U_{t}^{T} & =\left[\Delta U_{t}^{T}(t) \ldots \ldots \ldots \ldots . \Delta U_{t}^{T}\left(t+h_{p}-1\right)\right] \\
Y_{0}^{T} & =\left[y_{0}^{T}(t+1) \ldots \ldots \ldots \ldots y_{0}^{T}\left(t+h_{p}\right)\right]
\end{aligned}
$$

Note that $G^{j}\left(q^{-1}\right)=B\left(q^{-1}\right)\left[1-q^{-j} F^{j}\left(q^{-1}\right)\right] / A\left(q^{-1}\right) \Delta$ so that one way to computing $G^{j}$ is simply to consider the $Z$-transform plant's step-response and to take the first $j$ terms and therefore $g_{i}^{j}=g_{j}$ for $\mathrm{j}=0,1,2 \ldots<\mathrm{i}$ independent of the particular $\mathrm{G}$ polynomial[1].

The matrix $G$ is then lower-triangular of dimension $h_{p} \times h_{p}$ : 


$$
G=\left[\begin{array}{ccccc}
g_{0} & 0 & \ldots & \ldots & 0 \\
g_{1} & g_{0} & \ldots & \ldots & 0 \\
. & . . & \ldots & \ldots & . \\
\vdots & & & & \vdots \\
g_{h_{p}-1} & g_{h_{p}-2} & \ldots & \ldots & g_{0}
\end{array}\right]
$$

Note that if the plant dead time $d>1$ the first $d-1$ rows of the $G$ will be null, but if instead $h_{i}$ is assumed to be equal to $d$ the leading element is non-zero[1].

From the definitions above of the vectors and with:

$$
Y^{* T}=\left[y^{* T}(t+1) \ldots \ldots \ldots . . . y^{* T}\left(t+h_{p}\right)\right]
$$

The expectation of the cost-function of (4) can be written as follow:

$$
\begin{aligned}
& J\left(h_{i}, h_{p}, h_{c}, t\right)= \\
& \left(G \Delta U_{t}+Y_{0}-Y^{*}\right)^{T} R\left(G \Delta U_{t}+Y_{0}-Y^{*}\right) \\
& +\Delta U_{t} Q \Delta U_{t}^{T}
\end{aligned}
$$

The solution, $\Delta U_{t}$ minimizing the criterion can be explicitly found, using:

$$
\frac{\partial J}{\partial \Delta U_{t}}=0
$$

it follows that:

$$
\Delta U_{t}^{*}=\left(G^{T} G+Q\right)^{-1} G^{T} R\left(Y_{0}-Y^{*}\right)
$$

Note that the first element of $\Delta U_{t}^{*}$ is $\Delta u(t)$ so that the current control $\mathrm{u}(\mathrm{t})$ is given by:

$$
u(t)=u(t-1)+\left(G^{T} G+Q\right)^{-1} G^{T} R\left(Y_{0}-Y^{*}\right)
$$

- Case $2 h_{c}<h_{p}, h_{i}=1$

It is possible to reduce computational burden by imposing a constant control input vector after a fixed horizon $h_{c}$ $\Delta u(t+j-1)=0$ for $\left.\mathrm{j}>\mathrm{h}_{\mathrm{c}}\right)$

In this case the vector $\Delta U_{t}$ and the matrix $\mathrm{G}$ become:

$$
\begin{gathered}
\Delta U_{t}^{T}=\left[\Delta U_{t}^{T}(t) \ldots \ldots \ldots \ldots . \Delta U_{t}^{T}\left(t+h_{c}-1\right)\right] \\
G=\left[\begin{array}{ccccc}
g_{0} & 0 & \ldots & \ldots & 0 \\
g_{1} & g_{0} & \ldots & \ldots & 0 \\
. & \ldots & \ldots & \ldots & g_{0} \\
\vdots & & & & \vdots \\
g_{h_{p}-1} & g_{h_{p}-2} & \ldots & \ldots & g_{h_{p}-h_{c}}
\end{array}\right]
\end{gathered}
$$

\section{Adaptive Control Algorithm}

The adaptive controller which is proposed here is indirect controller. To estimate the unknown system parameters $\theta=\left(a_{1}, \ldots, \mathrm{a}_{\mathrm{na}}, b_{0}, \ldots, \mathrm{b}_{\mathrm{nb}}\right)^{T}$ the Recursive Least Squares (RLS) algorithm parameter estimates $\hat{\theta}_{t}$ are using for tuning of the Generalized Predictive Controller. Thus, the obtained Adaptive Generalized Predictive Controller generates the current control signal.

The following (RLS) algorithm has been using:

$$
\begin{gathered}
\hat{\theta}(t)=\hat{\theta}(t-1)+\frac{\varepsilon(t) \Delta \Phi(t-1) F(t-1)}{1+\Delta \Phi^{T}(t-1) F(t-1) \Delta \Phi(t-1)} \\
\varepsilon(t)=\Delta y(t-1)-\theta(t-1) \Delta \Phi(t-1) \\
F(t)=\frac{1}{\lambda_{1}(t)}\left[F(t-1)-\frac{\Delta \Phi(t-1) \Delta \Phi^{T}(t-1) F(t-1)}{\frac{\lambda_{1}(t)}{\lambda_{2}(t)}+\Delta \Phi^{T}(t-1) F(t-1) \Delta \Phi(t-1)}\right]
\end{gathered}
$$

Where,

$$
\begin{gathered}
\hat{\theta}(t)=\left[\hat{a}_{1}, \ldots, \hat{a}_{\mathrm{na}}, \hat{b}_{0}, \ldots, \hat{b}_{\mathrm{nb}}\right]^{T} \\
\Phi(t)=\left[y^{T}(\mathrm{t}-1), \ldots, y^{T}(\mathrm{t}-\mathrm{na}), u^{T}(\mathrm{t}-1), \ldots, u^{T}\left(\mathrm{t}-\mathrm{n}_{\mathrm{b}}\right)\right]^{T}
\end{gathered}
$$

Where:

$\varepsilon(t)$ is the estimation error

$\Phi(t)$ is the vector of data input-output

$\mathrm{F}(\mathrm{t})$ is the adaptation gain

$\lambda_{1}(t)$ and $\lambda_{2}(t)$ represents forgotten factors.

\section{Simulation and Discussion}

In order to illustrate the behavior of the above presented Adaptive Generalized Predictive Control, the simulation results of the heat exchanger pilot plant model obtained by using on-line identification technique[11], are given. The model is chosen as follows:

$$
\begin{aligned}
& y(t)-1.5326 y(t-1)+0.8174 y(t-2)-0.2051 y(t-3)= \\
& -0.0254 u(t-1)+0.0451 u(t-2)-0.0609 u(t-3)
\end{aligned}
$$

Several experiments have been carried out to determine a suitable control model order an appropriate sample time for control. A tried order model (na $=3, n b=2$, delay $=0)$ sampled at 1 second gave a reasonable description of a heat exchanger using on-line pilot plant dynamics.

The simulation has been done with respect to the following considerations:

- The sampling time $\mathrm{T}=1$

- The plant model structure $n a=3, n b=2$ and delay $=0$

- The reference is chosen as a square wave

- The polynomial $C\left(q^{-1}\right)$ chosen as $C\left(q^{-1}\right)=1$

- The initial covariance matrix $F(0):=10^{\wedge}$

\subsection{Case 1: Simulation in Noise Absence Conditions}

\subsection{Case 2: Simulation In Noise Presence Conditions}

In the figures above, it can be observed the comparative results between Generalized Predictive Control and Adaptive Generalized Predictive Control, the same variation of parameters was applied. 
The control performances of the Generalized Predictive Control and Adaptive Generalized Predictive Control with reference to set point changes are shown in Figures $(2,3,4$, and 5): the Adaptive Generalized Predictive Control have the same response as the Generalized Predictive Control with less oscillation.

The robustness of the control schemes to noises affecting the output has also been tested. Figures $(7,8,9$ and 10) refer

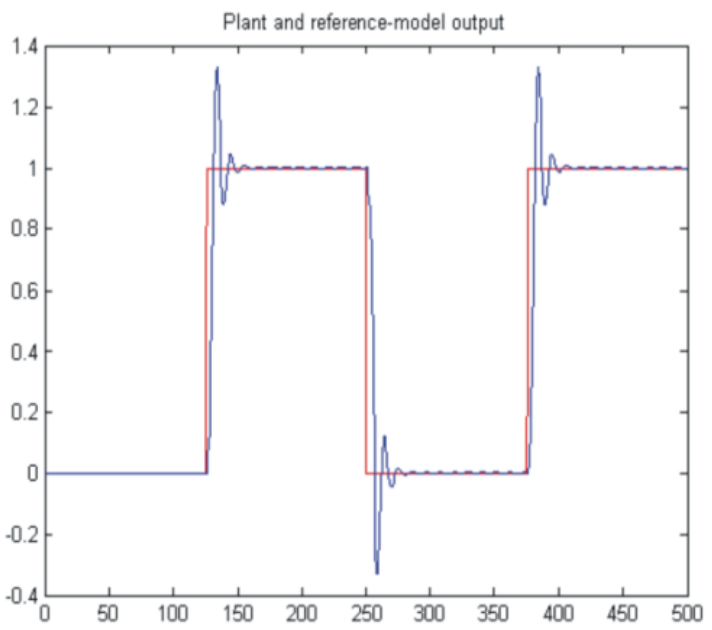

Figure 2. Plant output in noise absence conditions: GPC Control

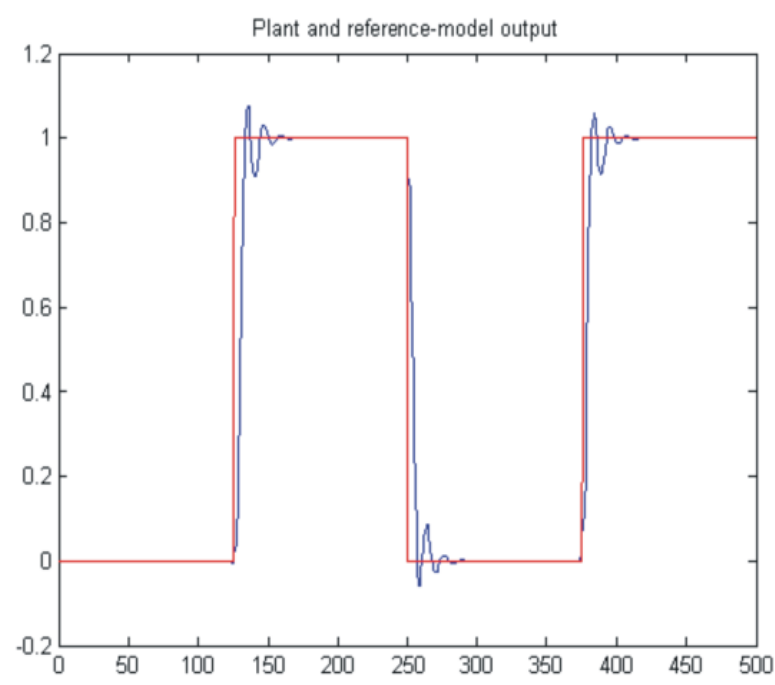

Figure 4. Plant output in noise absence conditions: Adaptive predictive control to the case of white output noise $\left(\sigma^{2}=0.01\right)$ : in addition to previous considerations which are still fulfilled, it can be observed that the Adaptive Generalized Predictive Control shows better characteristics concerning the variances of the plant output and the control input.

The behavior of the model's parameters is shown in figures 6 and 11 .

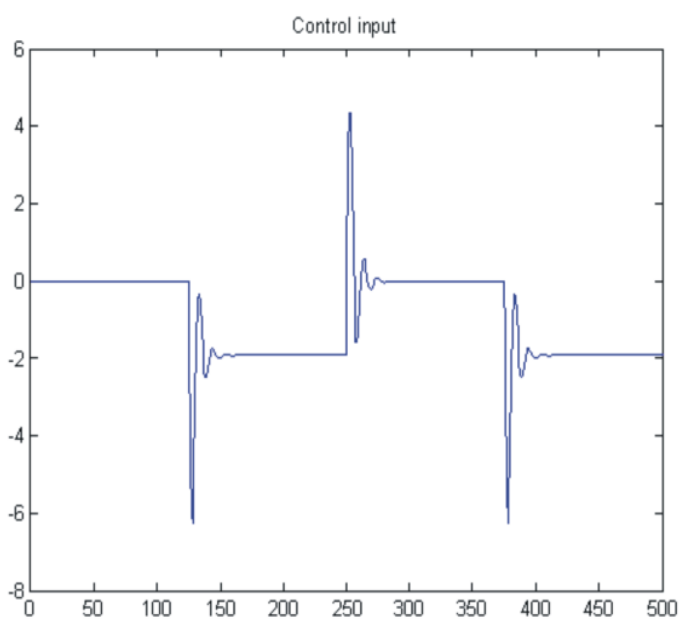

Figure 3. Control input in noise absence conditions: GPC Control

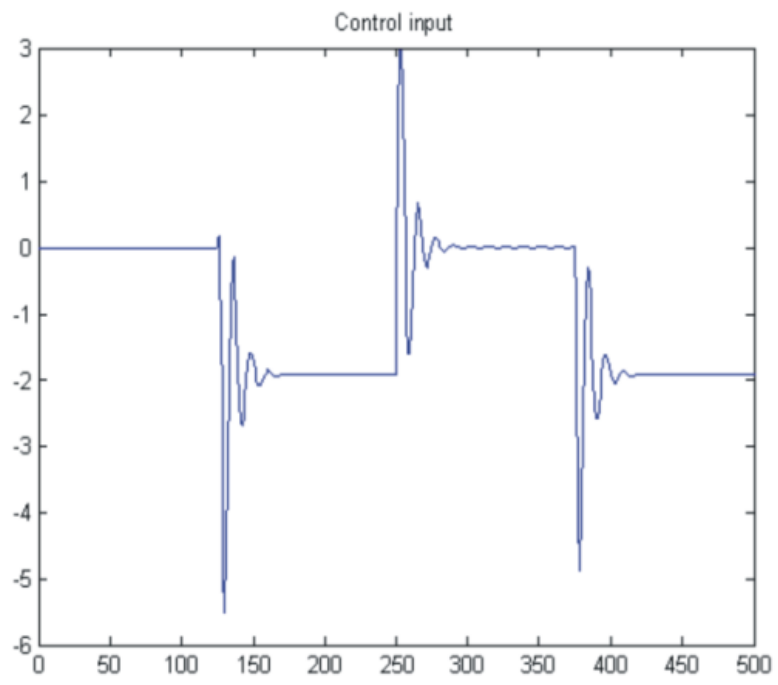

Figure 5. Input control in noise absence conditions: Adaptive predictive control 

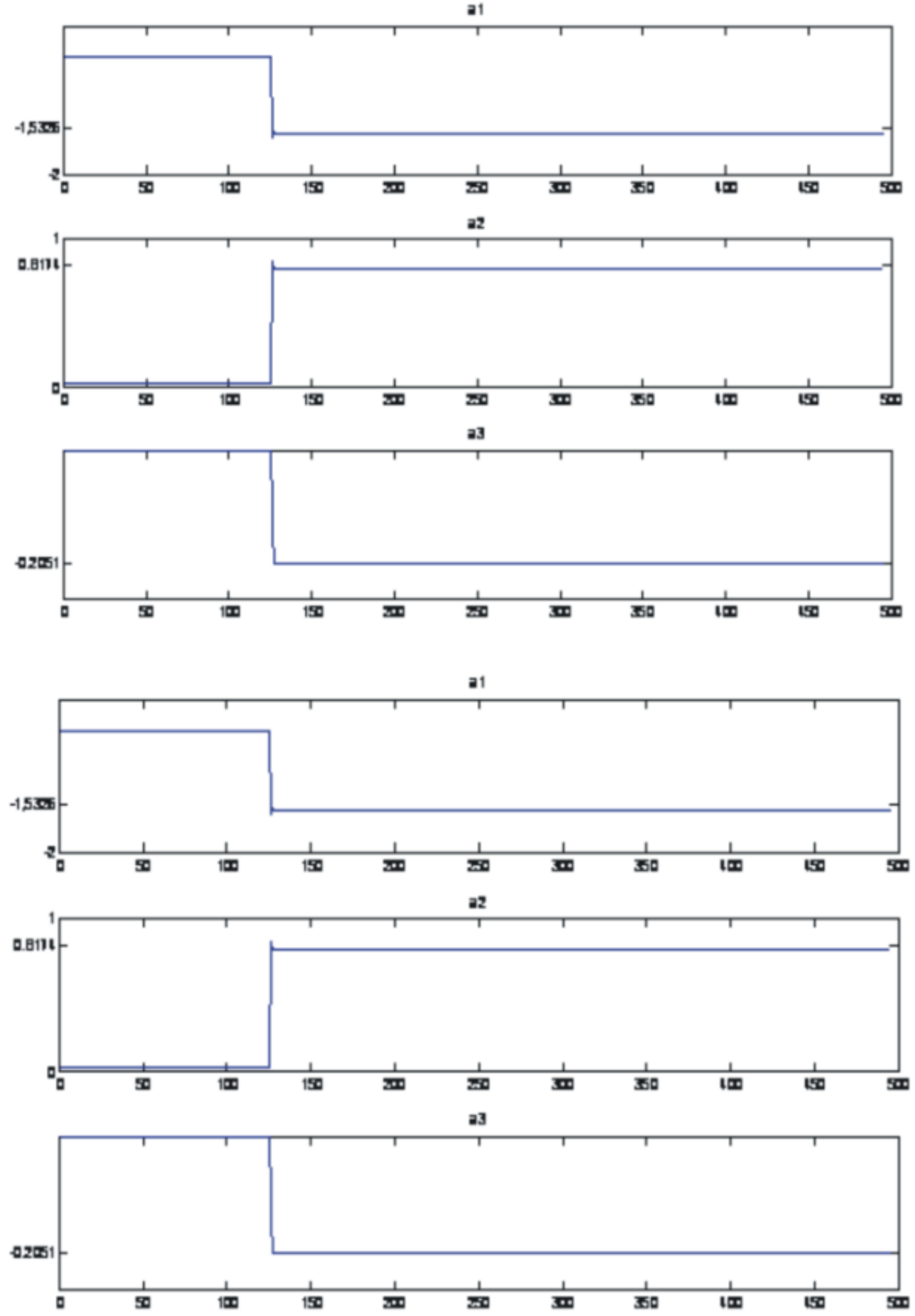

Figure 6. Tuned parameters in noise absence conditions 


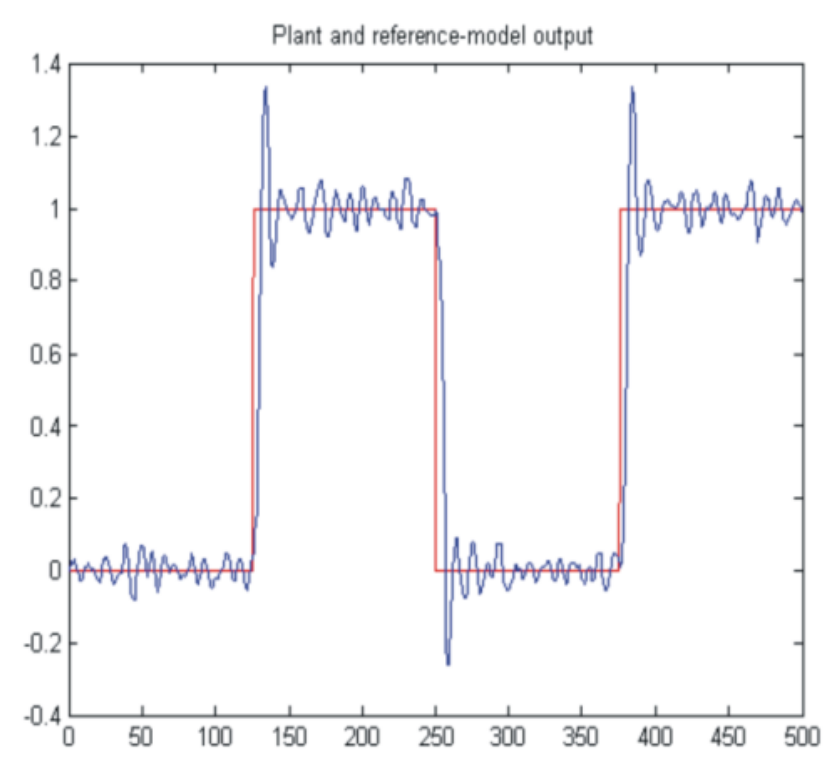

Figure 7. Plant output in noise case: GPC Control

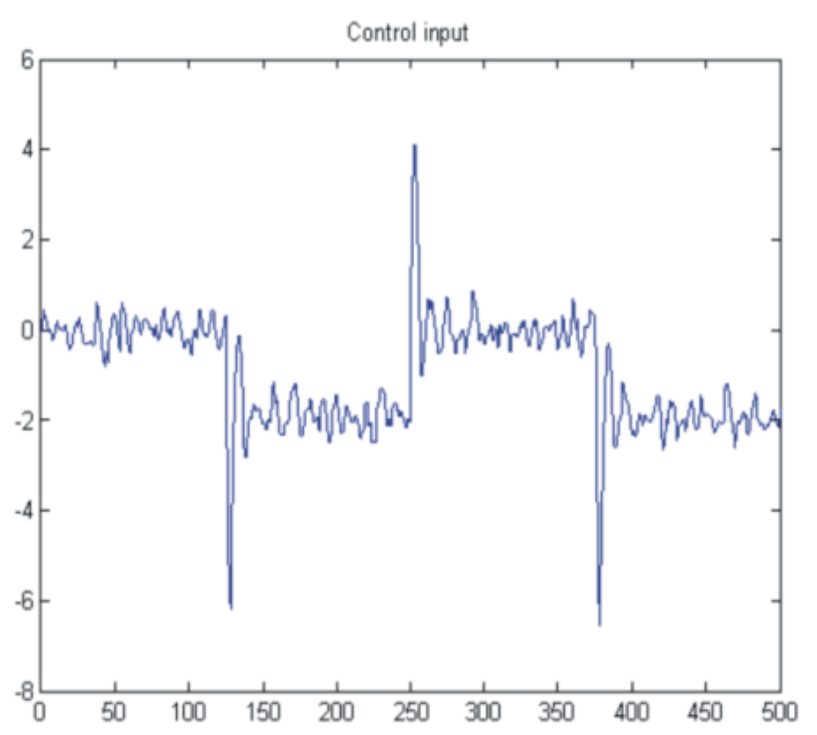

Figure 8. Control input in noise case: GPC Control

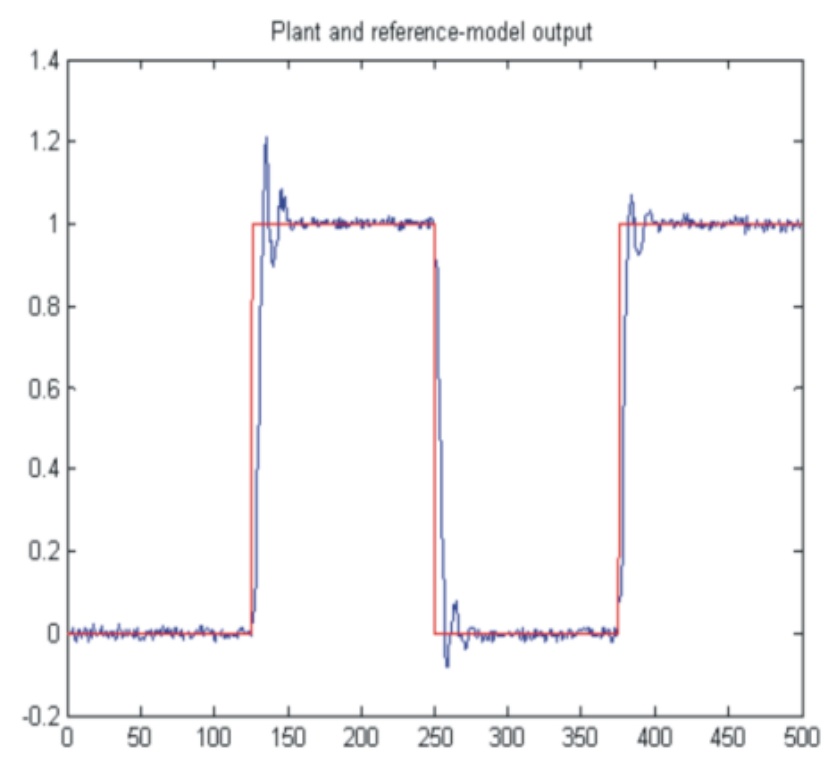

Figure 9. Plant output in noise case: Adaptive predictive control

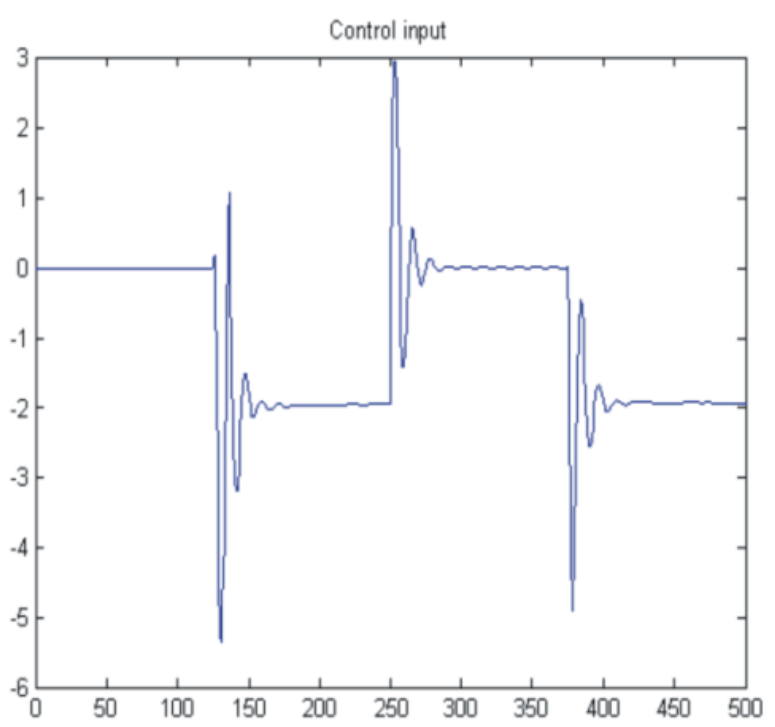

Figure 10. Input control in noise case: Adaptive predictive control 

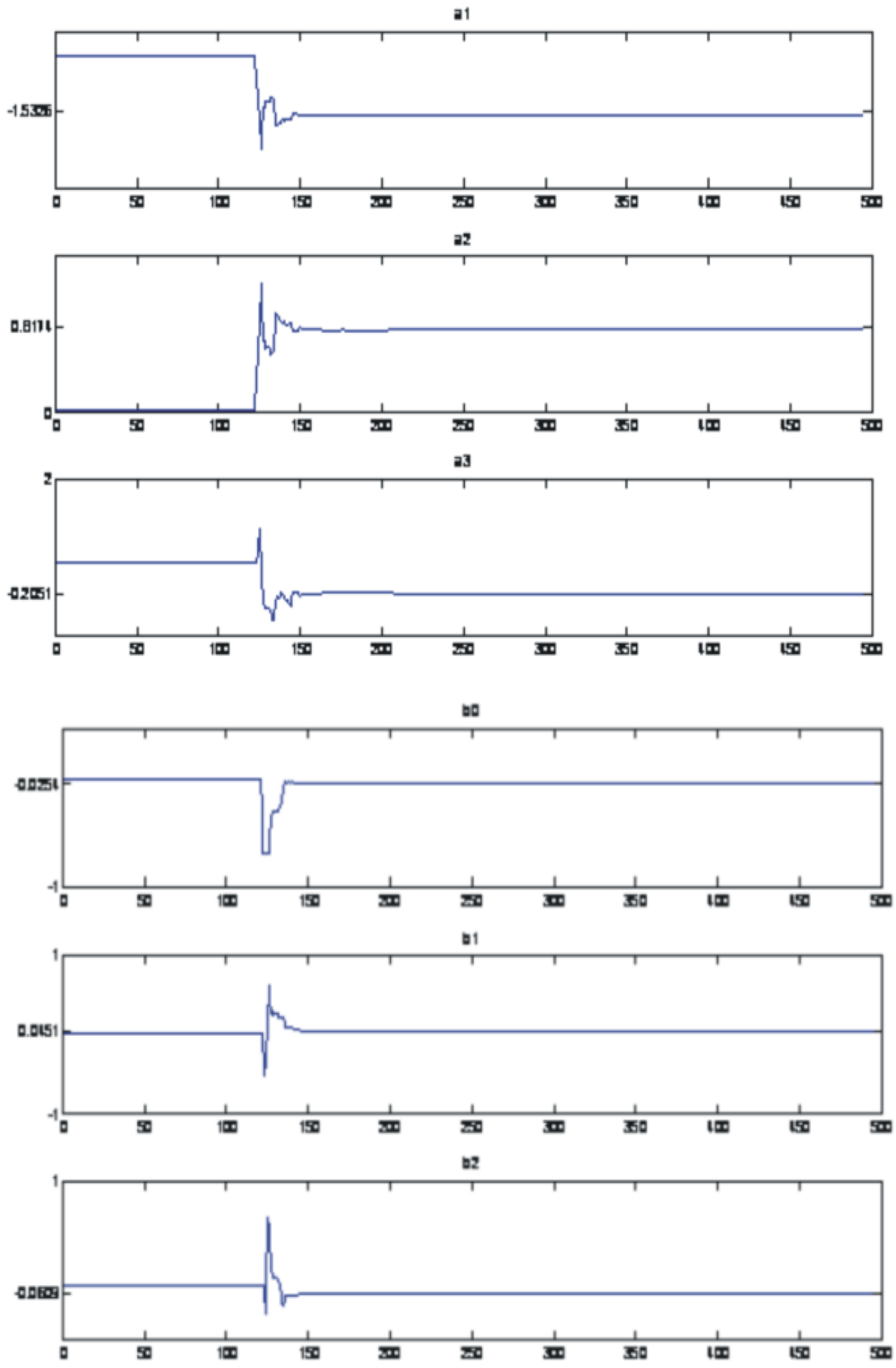

Figure 11. Tuned parameters in noise case 


\section{Conclusions}

In this paper, an Adaptive Generalized Predictive Control strategy is applied to a heat exchanger pilot plant. It has proved that, even with important variations of the plant output, the developed adaptive structure maintains a high level of performances, in terms of tracking, disturbance robustness and overshoot, cancellation of oscillation.

\section{REFERENCES}

[1] D. W. Clarke, C. Mohtadi, and P. S. Tuffs, "Generalized Predictive Control-part I. The Basic Algorithm", Automatica. Vol. 23, No. 2, pp. 137-148, 1987.

[2] D. W. Clarke, C. Mohtadi, and P. S. Tuffs, "Generalized Predictive Control-part II. Extentions and Interpretations", Automatica. Vol. 23, No. 2, pp. 149-160, 1987.

[3] P. Pivonka and P. Nepevny, "Generalized Predictive Control with Adaptive Model Based on Neural Networks", in Proceedings of the 6th Wseas Int. Conf. on Neural Networks, Lisbon, Portugal, pp. 1-4, June 16-18, 2005.

[4] C. Bordons and E.F. Camacho, "A Generalized Predictive Controller for a wide class of industrial Processes", Vol 6, No. 2, pp 372-387, 1998.

[5] N. Sepehri and G. Wu, "Experimental evaluation of Generalized Predictive Control Applied to a Hydraulic Actuator ", Robotica, Vol. 16, pp. 463-474, 1998.

[6] S. Chidrawar and B. Patre, "Generalized Predictive Control and Neural Generalized Predictive Control", Leonardo Journal of Sciences, Issue 13, PP. 133-152, July-December, 2008.
[7] Proceedings of the 6th Wseas Int. Conf. on Neural Networks, Lisbon, Portugal, pp. 1-4, June 16-18, 2005.

[8] J.K. Gruber, D.R. Ramirez, T. Alamo, C. Bordons, E.F. Camacho, "Min-Max Model Predictive Control of a Pilot Plant", Proceedings of the 2008 American Control Conference, Westin Seattle Hotel, Seattle, Washington, USA, pp. 1115-1120, June 11-13, 2008.

[9] M. Jalili-Kharaajoo and B. N. Araabi, "Neural Network Based Predictive Control of a Heat Eexchanger Nonlinear Process", Journal of electrical \& electronics engeneering. Vol. 4, No. 2, pp. 1219-1226, 2004.

[10] D. Bonivento, P. Castaldi and D. Mirotta, "Predictive Control vs PID Control of an Industrial Heat Exchanger".

[11] Totok R. Biyanto, M. Ramasamy, H. Zabiri, "Modeling Heat Exchanger Using Neural Networks", Proceedings of the International conference on Intelligent and Advanced Systems, pp. 120-124, 2007.

[12] U. Baur and R. Izermann, "On-line identifiction of a heat exchanger with process computer case stady", Automatica. Vol. 13, pp. 487-496, 1977.

[13] Totok R. Biyanto, M. Ramasamy, H. Zabiri, "Modeling Heat Exchanger Using Neural Networks", Proceedings of the International conference on Intelligent and Advanced Systems, pp. 120-124, 2007.

[14] Z. Zidane, M. Ait Lafkih, M. Ramzi, "Adaptive Generalized Predictive Control of a Heat Exchanger Pilot Plant", Proceedings of the 2011 International conference on Multimedia Computing and Systems, April 7-9, 2011.

[15] Z. Zidane, M. Ait Lafkih, M. Ramzi, "Adaptive Control of a Heat Exchanger", Proceedings of the International congress on Numerical Analysis and Scientific Computing With Applications in Sciences and Engineering Settat (Morocco), April 19-20, 2011. 